Chapter 2

\title{
Cognitive Processes and Biases in Obsessive-Compulsive Disorder
}

\author{
Elisa Krackow, Sarah Nunley and Pamela Tessier \\ Additional information is available at the end of the chapter \\ http://dx.doi.org/10.5772/58406
}

\section{Introduction}

Empirically-supported treatments for obsessive-compulsive disorder (OCD) emphasize the behavioral principles of exposure and response prevention [1]. However, theory and data suggest that cognitive components play a substantial role in the etiology and maintenance of OCD. This chapter will review cognitive theories and models of OCD, and cognitive processes in OCD including thought-action-fusion, inferential confusion, reality monitoring, cognitive inhibition, and memory and attentional biases. A comprehensive treatment that draws on both the cognitive literature reviewed herein plus exposure and response prevention is proposed.

\section{General cognitive theories of OCD}

The seminal cognitive theory by Rachman describes a diathesis-stress model in which an individual's history of being reared in an environment where strong values are placed upon the person's thoughts increases vulnerability to OCD [2]. Current levels of stress and negative affect increase the likelihood of obsessions which tend to be elicited by cues in the external environment [3]. Learning history then increases the likelihood of the obsessions being appraised as significant or meaningful. The appraisal of the obsessions as significant represents a cognitive bias because without a cognitive vulnerability towards $O C D$, credibility would not be given to the thoughts that become obsessions. These processes are more likely to occur in individuals with pre-existing trait anxiety. For example, an anxious person may read a story in a newspaper about an electrical house fire and then obsess over the possibility of accidentally setting an electrical fire. 
It is the above combination of factors that lays the groundwork for obsessions, coupled with other specific cognitive processes and biases which are about to be discussed. Overestimating the probability of the occurrence of negative events can occur if one believes that one is responsible in a situation. For example, a person may believe that "a child is more likely to have an accident if I am babysitting than if someone else is babysitting." Relatedly, Rachman discusses the cognitive process of Thought-Action Fusion (TAF) [2]. TAF is a cognitive distortion representing a moral component and an event probability component. A moral reasoning error is made when an individual has a thought about an action and then appraises that thought as being equivalent or almost equivalent to actually having engaged in the action itself. In TAF, the probability of an event occurring can be perceived as increasing simply by having a thought about the event. Both the moral reasoning and event probability components fuse thoughts and actions. TAF is increased by the overestimation of attributions of responsibility to the self for events, which represents a cognitive distortion. It should be noted that although TAF is a component of Rachman's cognitive theory, it is described in other literature as a separate theory [4].

Salkovskis (1985) proposed a second cognitive theory of OCD [5]. As Salkovskis points out, the components of this theory have substantial overlap with Rachman's previously described theory. According to this theory, obsessions can be cued via stimuli in the environment. Cuing of obsessions is aversive to the individual and therefore avoidance measures are taken. The person may or may not recognize the obsessional thought to be irrational. If the thought does not have negative ramifications for the person, this cognitive chain of events will end. If negative ramifications are present, automatic thoughts, similar to Beck's conceptualization of automatic thoughts will occur [6]. Automatic thoughts are more likely to occur if the current mood state is negative. These thoughts interact with a set of assumptions, including but not limited to, thought equals action, increased sense of responsibility for events, beliefs about the need to control thoughts, and self blame for events. These negative thoughts then influence mood which leads to attempts to reduce distress by engaging in compulsions. Negative reinforcement occurs when the distress is reduced lending credibility to the negative automatic thoughts. Co-occurring depressed mood can also play a role via several mechanisms. The first mechanism increases the dysfunctional beliefs via increasing the stimuli that may cue an intrusive thought that could become an obsession. This can then create a transactional process by which mood increases negative automatic thoughts and in turn, these thoughts then can increase negative mood.

Rachman proposed a specific cognitive theory of the compulsive checking subtype of OCD [7]. According to this theory, individuals with this subtype of OCD have an increased belief in self-responsibility for maintaining the safety of self and others. The increased sense of responsibility comes from an unrealistic appraisal of the probability of harm. Repeated checking serves the function of increasing certainty that any possible threat will be alleviated. Given that certainty is improbable, checking continues. Repeated checking results in memories of repeated events becoming fuzzy, and therefore confidence in memory as to whether checking has or has not occurred is decreased. That is, checking behavior temporarily reduces 
the anxiety via engaging in the compulsive behavior itself or via negatively reinforcing cognitions.

\section{Specific cognitive processes in OCD}

Thought-action fusion and OCD. Thought-action fusion is a component of OCD in the two previously discussed theories [2,5]. Researchers have developed assessment measures of TAF which have resulted in empirical findings supporting TAF as a process in OCD. Most commonly, TAF is measured using the Thought-Action-Fusion questionnaire, a 19-item questionnaire composed of moral TAF, likelihood-self TAF, and likelihood-other TAF scales [8]. Accordingly, the two factors of moral and likelihood TAF accounted for $71 \%$ of the variance. Internal consistency ranged from.88 to.96, and the TAF converged as expected with the Maudsley Obessive Compulsive Inventory. As expected, an OCD sample exhibited higher TAF scores on all three scales than did two pathology-absent adult comparison samples. TAF is not specific to patients with OCD but rather occurs with other anxiety disorders [9].

Researchers have examined whether TAF is more common to depression than to OCD due to high negative affect being characteristic of depression [10]. Drawing on Rachman's model, Abramowitz and colleagues theorized that negative affect mediates the relationship between TAF and OCD [2]. Their results supported this view. Interestingly, this study also found that the likelihood component but not the moral component of TAF is present in individuals with OCD. Researchers have further specified the relationship between these variables [11]. They examined the relation between TAF, schizotypal traits (magical thinking), OCD symptoms, general anxiety, and depression. Schizotypal traits were deemed important to include because nearly half of people with OCD have schizotypal personality characteristics. Among those characteristics are magical thinking, which the authors note may be associated with cognitive biases and therefore increased vulnerability to OCD. Schizotypical traits were associated with likelihood TAF such that the relation between TAF and OCD disappeared when schizotypal traits were controlled. No significant relation was found between OCD and moral TAF. In a third investigation, other investigators examined TAF, self-doubt regarding the belief that the compulsion will fix the problem, and cognitive control when obsession related beliefs emerge in youth with OCD, youth with another anxiety disorder, and a psychopathology-absent comparison group [12]. They found that higher likelihood TAF occurs in youth with OCD compared to youth without psychopathology, with no significant differences between the OCD and other-anxiety disorder groups, but self-doubt does not differ between the OCD and control group. Moral TAF was not measured in this study.

In sum, people with OCD tend to make cognitive errors in their thinking with regard to the fusion of thoughts and actions. This tends to occur in the following two ways: 1). Having a thought can be viewed as being as "bad" as having completed the action (moral TAF) and 2). thinking about the events increases the perception that the event will occur (likelihood TAF). The evidence appears to be slightly stronger for likelihood TAF than for moral TAF, but moral TAF was not measured in all studies examining this construct. Some evidence suggests that TAF is not specific to OCD but instead also occurs in anxiety disorders in general. 
Inferential Confusion and OCD. A concept related to TAF is "inferential confusion" or the process of confusing imagined events and actually occurring events. According to this view people with OCD mistake the imagined negative event for a real event, such that the imagined event becomes more realistic creating the belief that the event has an increased probability of occurrence [13]. Devaluation of information taken in by the senses (e.g., the tendency to believe something despite the available evidence to the naked eye) and inferential reasoning errors combine to make conditions ripe for the confusion of real and imagined events [14]. A "crossover point" can be identified in these individuals in which obsessions begin and can be identified [13]. Compulsions result from the obsessions as attempts to modify the probability of the event occurrence. Engaging in compulsions gives credibility to the imagined event. A specific type of inferential confusion can occur in which thinking a particular thought makes it likely that a person will act on that thought. Therefore, similar to TAF, a thought is equivalent to a personal action.

Support for inferential confusion theory comes from a follow-up study in which participants with OCD were given questionnaires measuring inferential confusion and obsessional thinking [15]. Analyses showed strong overlap between inferential confusion and common obsessions such as contamination and harm, and compulsions, such as washing. Even when other variables were controlled (e.g., overestimation of threat and general anxiety), the relationship between inferential confusion and obsessions/compulsions remained. Additional data show that when inferential confusion improves (as measured by a specific paper and pencil questionnaire of inferential confusion), OCD symptoms improve [16].

In a nonclinical sample, schizotypal symptoms (delusional thinking, perceptual disturbances, living in an inner world) and inferential confusion predicted obsessive compulsive behavior, with the majority of the predictive validity coming from the perceptual disturbances subscale of the schizotypal measure (22\% of the variance) [17]. Inferential confusion explained $5 \%$ of the variance. The authors note that people with OCD typically do not have perceptual disturbances, but perceptual disturbances can be considered to be a product of inferential confusion.

Reality monitoring and intrusive imagery in OCD. Reality monitoring errors occur when people confuse real and imagined events [18]. It has been suggested that poorer reality monitoring ability in OCD individuals due to intrusive imagery present in OCD [19]. That is, with the occurrence of repeated involuntary images, those images seem real which leads participants to confuse them with reality. Indeed there is a large body of literature now showing that imagination leads people to believe in events that never occurred (see [20] for a review). Therefore, other researchers examined reality monitoring ability for actions in an inpatient group with OCD and a yoked nonanxious control group [21]. In this study, participants were given sequences of 6 actions and instructed to either perform an action or imagine performing an action. Action sequences were either anxiety provoking or neutral. There were no significant differences in ability to identify imagined versus performed actions between the groups. Results did not differ as a function of whether the action sequences were anxiety-provoking or neutral. However, people with OCD had less confidence in their reality monitoring ability. 
Relatedly, deficits in reality monitoring ability have been shown to occur in people with subclinical OCD symptoms (specifically checking behaviors) compared to people without OCD symptoms [22]. To document this effect, all participants were read a list of actions. They were then asked to perform an action, observe an action being performed on a videotape, or write down a never performed action that was read to them by an experimenter. Following this task, participants were then asked to generate a written list of all actions they could recall, and then reality monitor by indicating whether they had performed, observed, or written each action they had recalled. People with OCD symptoms recalled fewer of the performed, written, and observed actions. In addition, they miscategorized (made more reality monitoring errors) whether they performed an action, wrote down the action, or observed the action being performed.

Additional data suggest that intrusive imagery related to the experience of adverse life events is a component of OCD at least in some patients. In an inpatient OCD sample, $81 \%$ of 34 patients reported intrusive images directly from or related to adverse life events [23]. Interestingly, none of the patients met co-morbid criteria for PTSD. OCD patients with intrusive images had higher levels of general anxiety and more OCD symptoms than OCD patients who did not have images. However, the number of patients without images was small $(n=7)$. Consistent with Rachman's previously discussed theory, patients who reported imagery felt greater responsibility for events than those who did not report images, although the origins of this increased responsibility are unclear.

Other reports of intrusive images in OCD have been found in the literature [24]. Therefore, Rachman correlated a single item measuring intrusive images with mental contamination (feeling dirty inside) [24]. This item correlated approximately.54 with other measures of OCD. Based on analyses from another archival dataset, Rachman reported that mental imagery items correlated.34-.445 with other measures of OCD [24]. Therefore, it has been suggested that imagery rescripting of distressing events might be an intervention appropriate for these individuals, but also point out that it is unknown whether this would decrease OCD symptoms given the lack of current empirical data for this intervention for OCD [23].

In sum, the evidence suggests that imagery can be an important in the treatment of people with OCD who experience intrusive images from negative life events. More broadly, imagery plays a role in the development OCD. Imagery acts as a mechanism by which obsessions come to seem more realistic thereby increasing the perception of the likelihood of occurrence. However, specific tests of reality monitoring ability do not always show differences in the performance of people with and without OCD. Methodological issues, including the timing of memory tests (i.e., lack of a substantial delay between exposure to the stimuli and memory test), may have influenced the results of existing studies. Therefore, further research is needed.

\section{Memory biases in OCD}

Negative memory biases have been theorized to play a role in psychological disorders, particularly anxiety. According to Beck, negative schemata formed in childhood render an 
individual susceptible to drawing attention towards threatening stimuli which is theorized to positively impact memory for these stimuli [6]. These schemata are activated when ambiguous or anxiety-provoking stimuli are encountered and predispose a person towards an anxious interpretation of events. A more specific model of how anxiety influences the allocation of attention and memory has been developed [25]. Specifically, when a stimulus is encountered in the environment, it is mapped onto existing schemata and a decision is made as to the threat level of the stimulus. When stimuli are appraised as threatening, they receive further cognitive resources allocated towards them. That is, attention is drawn towards those stimuli, particularly in people high in trait anxiety. The opposite cognitive allocation or drawing attention away from the stimulus occurs in people low in trait anxiety which serves the function of not allocating unnecessary cognitive resources in the case of stimuli that do not warrant attention. Williams and colleagues discuss how the allocation of attention is a first step towards impacting or improving memory for those threatening stimuli [25].

Despite these theories, the rationale for studies examining memory biases in OCD focuses on studying phenomenon that have been observed in clinical settings rather than theory-based predictions. For example, because it has been noted that people with OCD express doubt regarding whether they have performed a particular behavior (e.g., checked whether they turned the stove off) which might lead to repeating the compulsion. One study compared people with the contamination subtype of OCD to both anxious controls to an undergraduate sample ( $n=10$ per group in the OCD and anxious control conditions) [26]. In this study, participants watched an experimenter touch objects with a "dirty" tissue (i.e., contaminate) or touch objects with a "clean" tissue (i.e., not contaminate). Memory was tested immediately following the task. People with OCD remembered "dirty" objects better. This pattern of findings did not hold for both the anxious control group or the undergraduate comparison sample. It should be noted that items were scored only for correct recall in this study. Analyses did not include errors in recall.

Other researchers replicated the above study [26] using a sample that included different control groups and included an analysis of recall errors [27]. The study included 16 OCD washers, 16 OCD checkers, 16 controls with anxiety (specific phobia), 16 in a no-pathology comparison group. Participants entered a room with 50 items which they then observed the experimenter touch with a tissue (prevents contamination) or bare hand (brings into contact with contamination). Participants were asked to recall the items 50 minutes later by first generating a written list of all items they recalled. For the recalled items only, they were then asked to recall whether the experimenter touched each item with tissue or bare hands. No significant group differences emerged in total number of items recalled in free recall. There was a difference in recall of whether the object was touched by the experimenter with tissue vs. hand. OCD washers showed superior accurate recall as to whether the objects were touched by bare hands. The anxious control group showed superior recall for objects touched by a tissue. These results suggest superior memory in OCD washers for "contaminated" items.

Another study examined whether differences in recall existed between an OCD sample and control sample as a function of the anxiety provoking properties of the to-be-remembered experienced versus imagined actions [21]. Participants had a booklet with 6 actions listed. They 
were asked to perform those actions. They were then taken to a room and were asked to either imagine or perform a set of 20 actions. They were then asked to state whether only the last item was imagined or performed. Following this they were asked for a series of ratings of vividness, confidence, desire for vividness, and satisfaction with memories. Results showed no significant difference in reality monitoring performance across anxiety provoking and nonanxiety provoking stimuli, but a significant difference in desire for vividness emerged and was greater in the OCD group. No significant differences were found on the other 3 ratings. However, the sample size in this study was small and may not have been sufficient to detect significance ( $N=19 ; 12$ OCD, 7 controls).

McNally and colleagues examined directed forgetting in OCD patients and controls [28]. Participants were presented with words of a negative, positive, or neutral valence followed by instructions directing them to remember or forget the just-presented stimuli. OCD patients had a more difficult time forgetting negative words than did comparison participants. However, it should be noted that a small portion of the words seemed to be directly related to specific types of OCD (contamination) and it is unclear whether the results would hold if these items were removed. It would be important to compare an OCD relevant negative list to another non-OCD related negative list to determine whether the above findings hold.

Another study examined memory as a function of experimenter induced sense of responsibility [29]. The authors note that people with OCD report a strong sense of responsibility for outcomes including correctness of their checking or lack of checking behavior. For example, one might think, "If I didn't look at the stove carefully to see whether it was off, it might still be on and the house may catch on fire." Following a baseline check in their homes to induce anxiety, the researchers had participants with OCD (all handwashers $N=11$ ) complete their typical checking behaviors (e.g., check to make sure the stove is off) in their homes under conditions of low and high responsibility. In the high responsibility condition, participants were told that they were responsible for completing the check without assistance and were responsible for the accuracy of the check. They were asked to sign a contract to this effect agreeing to these terms. In the low responsibility condition, participants signed a contract that attributed the responsibility of the check to the research assistant. Participants completed a memory interview immediately after the checks and provided confidence ratings for their responses to the interview questions. The memory interview included memory for specific aspects of the check that might have been perceived as threatening ("What was the final state of the thermostat?" (Radomsky et al., 2001, p. 817) [29] as well as nonthreatening aspects of the check ("Did I (the experimenter) cough during the check?) (Radomsky et al., 2001, p. 817) [29]. In the next phase of the study, all participants returned to the lab a week later and watched videos of both checks and then completed the same memory interview and confidence ratings. This delayed phase represented a no responsibility phase. Results showed no overall differences in the amount remembered across the conditions, but under conditions of high responsibility, OCD handwashers recalled more threatening information than irrelevant information. This result did not hold under conditions of low responsibility. When the people who were originally in the high responsibility group were tested under conditions of no responsibility one week later, they no longer showed superior recall of threatening information. However, 
this result is confounded by methodological issues. That is, threatening actions were sometimes performed by the participant whereas nonthreatening actions were sometimes performed by the experimenter. There is now a large body of literature showing superior memory for participation versus observation [30].

One study measured explicit memory with a recognition task for previously heard or never heard sentences and confidence [31]. Implicit memory was assessed via participants' ratings of the volume of the previously heard or never heard sentences. There was no tendency for OCD participants to rate previously heard sentences as louder thereby demonstrating a lack of a superior implicit memory bias. As expected, OCD participants did not show an explicit memory bias; that is they did not exhibit superior recognition performance compared to control participants nor did they correctly reject never-presented sentences at higher rates than control participants. Instead, OCD patients rated all sentences as louder than matched nontreatment seeking controls suggesting what the authors referred to as a "perceptual deficit," or difficulty accurately perceiving the stimuli, rather than memory deficit, although the ramifications of this finding are unclear. Failure to find an explicit memory bias appeared to be due to high accuracy rates in both groups. Perhaps a longer test delay would have been beneficial in this study.

In sum, the data on memory biases in OCD show that the majority of studies fail to find evidence for such as bias. Numerous methodological issues in the above described studies exist, thereby precluding firm conclusions from being drawn. Small sample size is clearly one limitation in some studies examining this topic. It is also possible that delay of test was not long enough in some of the above studies for differences to emerge. In some studies, participants were informed prior to encoding that memory would be tested, thereby raising the possibility that rehearsal strategies were used following experimenter notification that a memory test would occur. Other methodological issues include failure to score incorrect recall and confounds regarding the effects of participation.

\section{Attentional bias in OCD - Theory and research}

According to theory, individuals are more vulnerable to emotional disorders when the level of emotion exceeds an individual's capacity to control that emotion [32]. Individuals with anxiety disorders are more likely to display selective attention to threatening stimuli and experience more difficulty disengaging once attention to threatening stimuli is activated. It is also the case that the threshold for appraising a stimulus as threatening is lower in people with anxiety disorders. Accordingly, attention towards threat is a combination of current level of state anxiety, level of threat appraisal, and ability to modulate attention. Mathews and MacLeod suggest that attentional bias plays a role in the development of anxiety disorders [32]. Although many studies show that people with anxiety disorders display an attentional bias, these data do not show that attentional bias causes anxiety disorders. Indirect support for the hypothesis that attention plays a causal role in anxiety comes from studies that demonstrate that creating cognitive bias impacts emotional state. It is important to acknowledge that to date 
there has not been a study conducted in which an attentional bias towards anxiety is created with the resulting outcome showing that anxiety increases. However, research does show that training away from an attentional bias reduces anxiety [33]. Attentional bias is important in anxiety disorders because attending to threatening information leads to behavioral avoidance of the anxiety provoking and does not allow for exposure to the feared stimuli which would reduce the anxiety in the long term [34].

Several studies have examined whether attentional bias exists in patients with OCD. An early study presented patients with OCD and panic disorder, with OCD threat related words, panic threat related words, general threat related words, and neutral words within a stroop test [35]. There were no significant differences between OCD and panic patients with respect to correct responses on the stroop task. Response latencies also did not differ between panic and OCD participants. Note that longer latencies indicate greater interference or attentional bias. An earlier study presented within the same paper showed no significant differences between the panic and normal comparison groups. Taken together, these results argue against an attentional bias in OCD.

Using a new methodology that involved reaction time to investigate attentional bias, researchers compared people with OCD (predominantly checking behavior) to normal controls and found no significant differences in reaction time to checking related, paranoid theme related, or neutral words [36]. Another study found that an attentional bias was not present in people with OCD compared to normal controls when words were used in a stroop task, but attentional bias was present when pictures were used [37]. In contrast, a series of two studies using the dot-probe task demonstrated that people with OCD exhibited a contamination attentional bias and a general threat attentional bias [38]. However, the general threat attentional bias was equivalent to a high trait anxiety group thereby demonstrating that attentional bias is not specific to OCD. Neither the contamination attentional bias nor the general threat attentional bias was present in a normal control population.

Some evidence for attentional bias in people with an OCD washing compulsion as indicated by longer latencies to name words in a stroop task has been found, although all findings were not supportive of attentional bias [39]. The authors interpreted these results to suggest that the activation of fear in contamination $O C$ washers occurs and interferes with general information processing ability. Some evidence was found for general threat bias in OC nonwashers. Similarly, behavioral treatment of OCD, presumably exposure and response prevention, reduces fear sensitivity as measured by behavioral and physiological measures taken during a cognitive task [40].

Studies have investigated whether attentional bias could be modified in college students with subclinical OCD symptoms [33]. Specifically, the modification intervention focused on drawing participants' attention away from threatening words using a computerized task that displayed threat and neutral words at equal frequencies, but always drew participants' attention to the neutral words by replacing the neutral word with a probe. The modification intervention was administered after establishing that each participant exhibited an attentional bias. The modification intervention group was compared to a control group in which the probe replaced threatening or neutral words at equal frequencies. A behavioral avoidance task using 
items that participants were led to believe were contaminated served as the outcome measure. Participants who received attentional bias modification training engaged in less behavioral avoidance. They also showed less attentional bias during repeated trials of the attentional bias task administered immediately after bias modification training. An earlier study confirmed the presence of attentional bias in OCD but the bias was limited to the earlier portions of the task [41]. Bias was remediated via habituation in the later portions of the task.

Additional data show that people with OCD exhibit an attentional bias toward threatening information in general as opposed to only OCD related items [42]. These data further show that this bias is not due to other aspects of the stimuli that could account for the results, namely emotional arousal [43].

Relatedly, cognitive theories of OCD emphasize cognitive inhibition deficits in OCD, deficits in the allocation of attention via the inhibition of unwanted thoughts [44]. Experimental tests of cognitive inhibition show that people with OCD experience greater difficulty in ignoring previously presented stimuli when instructed to do so relative to people with anxiety disorders other than OCD [45]. Cognitive inhibition becomes further impaired as the complexity of the cognitive task increases [46].

In sum, a substantial amount of evidence exists for attentional bias in OCD. Importantly, attentional bias is not specific to OCD, but rather is present in anxiety disorders in general [47]. Nevertheless, existing studies show that when attentional bias is present in people with OCD, it can be modified, which can reduce behavioral avoidance of distressing stimuli. These data imply that clients should be assessed for attentional bias so that if present, this can be a focus of treatment.

\section{Conclusion}

\subsection{Do the extant data fit the etiological models of OCD?}

An examination of the data discussed herein reveals varying levels of support for the components of cognitive models of OCD described at the beginning of this chapter. Consistent support exists for inferential confusion which combines errors in reasoning with devaluation of information taken in by the senses and confusion between real and imagined events. The available data show both higher levels of inferential confusion in people with OCD and reduced inferential confusion following OCD treatment [15[16]. With regard to the role that the tendency to confuse real and imagined events plays in OCD (i.e., reality monitoring), the data are weaker $[21,22]$. However, it should be noted that reality monitoring ability is the only component of inferential confusion being examined in these studies. However, in inferential confusion processes, reasoning errors and the tendency to discount information taken in by the senses are factors that contribute to the tendency to make reality monitoring errors [14]. Nevertheless, a small amount of data do support the notion that imagery plays a role in the development or maintenance of OCD in people with OCD plus trauma histories [22,24]. However, further research should be conducted in this area. In addition, there is consistent support for the likelihood component of thought-action fusion $[8,10,11]$. That is, people with 
OCD are more apt to believe that having a thought increases the likelihood that the event contained in the thought is going to occur than people without OCD. Less support exists for the belief that having a thought that contains a moral transgression is the equivalent to having completed that transgression (moral component of TAF) [11]. With regard to memory biases in OCD, the data as a whole do not support the existence of memory biases in OCD [27]. However, it is the case that methodological issues in extant studies preclude firm conclusions from being drawn. Moreover, there are numerous studies showing support for the existence of an attentional bias in OCD [36,37,49,41,42]. Also, the data support attentional bias training in reducing OCD symptoms [33]. It should be noted that attentional bias is not a component of the theoretical models specific to OCD described herein, but the data support incorporating attentional bias into models of OCD.

It should be noted that research in this area is in its infancy. Although some but not other components of these models have been tested, extant research has not gone beyond asking the question of whether group differences exist between people with a diagnosis of OCD and various comparison or control groups. Longitudinal studies are needed to test these models holistically. In addition, other components of the models have not been examined. For example, there are no published data on family factors that lend themselves towards vulnerability to creating obsessions. In addition, cognitive appraisals of specific thoughts in people with OCD have not been studied. Moreover, data on the relations between life stress and OCD need to be collected. That is, stress levels in the year preceding the onset of OCD symptoms and overall history of life stress in people with OCD warrant study.

\subsection{Treatment implications of cognitive processes and biases in OCD}

The theories and data described throughout this chapter indicate that there is substantial room for cognitive components to make a significant contribution to OCD case conceptualization and treatment. Rachman's theory implies that psychoeducation about OCD should begin with presentation of the idea that obsessions are cued by stimuli in the environment [2]. Given that the environment is ripe with cues, clients can expect and should be prepared to expect that the obsessive thought will be cued. If clients are more distressed by obsessions than they are by compulsions, cognitive bias training modification could be employed at this point in the treatment. Modification training would be expected to decrease the obsessions because clients should be less likely to attend to and interpret environmental cues as being related to their obsessions. This would prevent the obsessions from being cued. If cued, clients can be taught that it is the actions that they now take when the obsession is present that are key. Clients should be taught to first, identify when the obsession has come to mind. It is at this point that the client needs to employ mechanisms to cope with the obsession, including cognitive restructuring. The therapist is advised to administer a measure of thought-action fusion such as the Thought-Action Fusion scale [8]. If scores are elevated in the clinical range, the clinician can introduce the idea of thought-action fusion and review each statement endorsed on the TAF as indicating that thoughts equal actions. In order to ward off inferential confusion, clients can be taught inferential reasoning skills that would be expected to reduce the tendency to make reasoning errors. In addition, clients can be taught reality monitoring skills to help remind themselves that a thought is not equal to an action. That is, clients can be taught to ask themselves, "Did I think X thought or did I perform X action?" [adapted from 48]. The therapist 
can then work with the client to restructure cognitions regarding the probability of events and responsibility for events. Given that the client has likely had a similar thought many times and not acted on it, what is the evidence that they will do so now? With regard to compulsions, clients should be taught about memory norms. First, repeated routine events tend not to be recalled [49]. Therefore, the fact that one cannot remember if one has turned off the stove is normal given the large number of checks performed; therefore, one's memory does not need to be confirmed. Skills from other therapies can be borrowed, such as distress tolerance skills from Dialetical Behavior Therapy [50]. That is, it is OK to feel distress in response to an obsession; one does not need to act on the distress by engaging in compulsions. Distress tolerance skills should be used as opposed to thought suppression skills because research shows that thought suppression can increase rather than decrease unwanted thoughts [51]. Similarly, behavioral activation can be employed so that the client engages in other activities besides rumination. Clients should receive psychoeducation about the normative progression of OCD symptoms. That is, they should expect that new compulsions may occur [52]. They can expect that the OCD will be worse in times of stress and can coincide with negative affect [2]. Consistent with behavioral theory, clients should be taught exposure and response prevention skills; family members that have become part of the OCD rituals should be participants in the therapy [53]. As in many empirically-supported treatments, relapse prevention skills should be taught and clinicians should be sure their clients are able to generalize these skills in order to deal with multiple obsessions and compulsions.

Although inclusion of cognitive components either in addition to exposure and response prevention or as a stand-alone treatment has not been found to add to the effectiveness of OCD treatment, existing treatment outcome studies have not included many of the components discussed in this section [54,55,56] [57]. Therefore, a more comprehensive treatment as proposed may yield more promising results.

\section{Author details}

Elisa Krackow*, Sarah Nunley and Pamela Tessier

*Address all correspondence to: elisa.krackow@mail.wvu.edu

Department of Psychology, West Virginia University, Morgantown, USA

\section{References}

[1] Abramowitz JS, Taylor S, McKay D. Potentials and limitations of cognitive treatments for obsessive-compulsive disorder. Cognitive Behaviour Therapy, 2005, 34(3) 140-147.

[2] Rachman SS. A cognitive theory of obsessions. Behaviour Research \& Therapy, 1997; 35(9) 793. 
[3] Julien D, O'Connor KP, Aardema F. Intrusions related to obsessive-compulsive disorder: A question of content or context? Journal of Clinical Psychology, 2009; 65(7) 709-722.

[4] Rassin E, Merckelbach H, Muris P, Schmidt H. The thought-action fusion scale: Further evidence for its reliability and validity. Behaviour Research \& Therapy, 2001; 39(5) 537.

[5] Salkovskis PM. Obessional-compulsive problem: A cognitive-behavioural analysis. Behaviour Research \& Therapy,1985; 23 571-583.

[6] Beck AT. Cognitive therapy and the emotional disorders. New York: International Universities Press; 1976.

[7] Rachman SS. A cognitive theory of compulsive checking. Behaviour Research \& Therapy, 2002; 40(6) 625.

[8] Shafran R, Thordarson MA, Rachman S. Thought-action fusion in obsessive-compulsive disorder. Journal of Anxiety Disorders. 1996; 10 379-391.

[9] Rassin, E., Diepstraten, P., Merckelbach, H., \& Muris, P.. Thought-action fusion and thought suppression in obsessive-compulsive disorder. Behaviour Research \& Therapy, 2001; 39(7), 757.

[10] Abramowitz JS, Whiteside SS, Lynam DD, Kalsy SS.. Is thought-action fusion specific to obsessive-compulsive disorder?: A mediating role of negative affect. Behaviour Research \& Therapy, 2003; 41(9) 1069. doi:10.1016/S0005-7967(02)00243-7

[11] Lee H, Cougle JR, Telch MJ. Thought-action fusion and its relationship to schizotypy and OCD symptoms. Behaviour Research \& Therapy, 2005; 43(1) 29-41. doi:10.1016/ j.brat.2003.11.002

[12] Barrett PM, Healy LJ. An examination of the cognitive processes involved in childhood obsessive-compulsive disorder. Behaviour Research \& Therapy, 2003; 41(3) 285. doi:10.1016/S0005-7967(02)00011-6

[13] O'Connor, K, Aardema F. Fusion or confusion in obsessive-compulsive disorder. Psychological Reports, 2003; 93(1) 227-232.

[14] Aardema, F, O'Connor, K., Emmelkamp, PMG, Marchand, A, \& Todorov, C. Inferential confusion in obsessive-compulsive disorder: The inferential confusion questionnaire. Behaviour Research and Therapy, (in press).

[15] Aardema F, O'Connor K, Emmelkamp P. Inferential confusion and obsessive beliefs in obsessive-compulsive disorder. Cognitive Behaviour Therapy, 2006. 35(3) 138-147. doi:10.1080/16506070600621922

[16] Aardema F, Emmelkamp PG, O'Connor KP. Inferential confusion, cognitive change and treatment outcome in Obsessive-Compulsive Disorder. Clinical Psychology \& Psychotherapy, 2005; 12(5) 337-345. doi:10.1002/cpp.464 
[17] Aardema F, Kleijer TR, Trihey M, O'Connor K. Processes of inference, schizotypal thinking, and obsessive-compulsive behaviour in a normal sample. Psychological Reports, 2006; 99(1) 213-220. doi:10.2466/PR0.99.1.213-220

[18] Johnson M, Raye CL. Reality monitoring. Psychological Review, 1981; 88(1) 67-85. doi: 10.1037/0033-295X.88.1.67

[19] Brown HD, Kosslyn SM, Breiter HC, Baer L, Jenike MA. Can patients with obsessivecomplusive disorder discriminate between percepts and mental images? A signal detection analysis. Journal of Abnormal Psychology, 1994; 103 445-454

[20] Krackow E, Rabenshorst M. Does the body know best? Psychophysiological reactivity to perceived versus imagines childhood events. Imagination, Cognition, and Personality. 2010; 30 133-145

[21] Constans JI, Foa EB, Franklin ME, Mathews A. Memory for actual and imagined events in OC checkers. Behaviour Research And Therapy,1995; 33(6) 665-671. doi: 10.1016/0005-7967(94)00095-2

[22] Rubenstein CS, Peynircioglu ZF, Chambless DL, Pigott TA. Memory in sub-clinical obsessive-compulsive checkers. Behaviour Research and Therapy, 1993, 31(8) 759-765. doi:10.1016/0005-7967(93)90006-G

[23] Speckens AE, Hackmann A, Ehlers A, Cuthbert B. Intrusive images and memories of earlier adverse events in patients with obsessive compulsive disorder. Journal of Behavior Therapy and Experimental Psychiatry 2007; 38 411-422.

[24] Rachman SS. Unwanted intrusive images in obsessive compulsive disorders. Journal of Behavior Therapy and Experimental Psychiatry,2007; 38 402-210.

[25] Williams JMG, Watts FN, MacLeod C, Mathews A. Cognitive psychology and emotional disorders. West Sussex, England: John Wiley \& Sons Ltd; 1997

[26] Radomsky AS, Rachman SS. Memory bias in obsessive-compulsive disorder (OCD). Behaviour Research \& Therapy, 1999; 37(7) 605.

[27] Ceschi G, Van der Linden M, Dunker D, Perroud A, Brédart S. Further exploration memory bias in compulsive washers. Behaviour Research \& Therapy, 2003; 41(6) 737. doi:10.1016/S0005-7967(03)00040-8

[28] Wilhelm S, McNally RJ, Baer L, Florin I. Directed forgetting in obsessive-compulsive disorder. Behaviour Research \& Therapy, 1996; 34(8) 633.

[29] Radomsky AS, Rachman SS, Hammond D. Memory bias, confidence and responsibility in compulsive checking. Behaviour Research \& Therapy, 2001; 39(7) 813.

[30] Tobey AE, Goodman GS. Children's eyewitness memory: Effects of participation and forensic context. Child Abuse \& Neglect, 1992; 16(6) 779-796. 
[31] Foa EB, Amir N, Gershuny B, Molnar C, Kozak MJ. Implicit and explicit memory in obsessive-compulsive disorder. Journal of Anxiety Disorders, 1997; 11(2) 119-129. doi:10.1016/S0887-6185(97)00001-7

[32] Mathews A, MacLeod C. Cognitive vulnerability to emotional disorders. Annual Review Clinical Psychology, 2005; 1 67-95.

[33] Najmi S, Amir N. The effect of attention training on a behavioral test of contamination fears in individuals with subclinical obsessive-compulsive symptoms. Journal of Abnormal Psychology, 2010; 119(1) 136-142. doi:10.1037/a0017549

[34] Tallis F. The neuropsychology of obsessive-compulsive disorder: A review and consideration of clinical implications. British Journal of Clinical Psychology, 1997; 36(1) 3-20.

[35] Kampman M, Keijsers GJ, Verbraak MM, Näring G, Hoogduin CL. The emotional stroop: a comparison of panic disorder patients, obsessive-compulsive patients, and normal controls, in two experiments. Journal of Anxiety Disorders, 2002; 16(4) 425-441.

[36] Moritz S, Von Mühlenen A. Investigation of an attentional bias for fear-related material in obsessive-compulsive checkers. Depression \& Anxiety (1091-4269), 2008; 25(3) 225-229. doi:10.1002/da.20294

[37] Moritz S, Fischer B, Hottenrott B, Kellner M, Fricke S, Randjbar S, \& Jelinek L. Words may not be enough! No increased emotional Stroop effect in obsessive-compulsive disorder. Behaviour Research \& Therapy, 2008; 46(9), 1101-1104. doi:10.1016/j.brat. 2008.05.005

[38] Tata PR, Leibowitz JA, Prunty MJ, Cameron M, Pickering AD. Attentional bias in obsessional compulsive disorder. Behaviour Research and Therapy, 1996; 34(1) 53-60. doi:10.1016/0005-7967(95)00041-U20.

[39] Foa EB, Ilai D, McCarthy PR, Shoyer B, Murdock T. Information processing in obsessive-compulsive disorder. Cognitive Therapy and Research, 1993; 17(2) 173-189. doi: 10.1007/BF01172964

[40] Foa EB, McNally RJ. Sensitivity to feared stimuli in obsessive-compulsives: A dichotic listening analysis. Cognitive Therapy and Research, 1986; 10(4) 477-485. doi: 10.1007/BF01173299

[41] Amir N, Najmi S, Morrison AS. Attenuation of attention bias in obsessive-compulsive disorder. Behaviour Research \& Therapy, 2009; 47(2) 153-157. doi:10.1016/j.brat. 2008.10.020

[42] Lavy EH, van Oppen P, van den Hout MA. Selective processing of emotional information in obsessive compulsive disorder. Behaviour Research and Therapy, 1994; 32(2) 243-246. doi:10.1016/0005-7967(94)90118-X 
[43] Matthew AM, Klug F. Emotionally and interference with color-naming in anxiety. Behaviour Research. \& Therapy, 1993; 23 563-69

[44] Beech AR, McManus D, Baylis GC, Tipper SP, Agar K. Individual differences in cognitive process towards an explanation of schizophrenic symptomatology. British Journal of Psychology, 1991; 82 417-426.

[45] Enright SJ, Beech AR. Reduced cognitive inhibition in obsessive-compulsive disorder. British Journal Of Clinical Psychology, 1993a; 32(1), 67-74. doi:10.1111/j. 2044-8260.1993.tb01028.x.

[46] Enright SJ, Beech AR. Further evidence of reduced cognitive inhibition in obsessivecompulsive disorder. Personality and Individual Differences, 1993b; 14(3), 387-395. doi:10.1016/0191-8869(93)90307-O.

[47] Mogg, K, Mathews, A, \& Eysenck, M. Attentional bias to threat in clinical anxiety states. Cognition \& Emotion,1992; 6 149-159.

[48] Giles JW, Gopnik A, Heyman GD. Source monitoring reduces the suggestibility of preschool children. Psychological Science, 2003; 13(3) 288-291. doi: $10.1111 / 1467-9280.00453$

[49] Hudson JA, Nelson K. Repeated encounters of a similar kind: Effects if familiarity on children's autobiographic memory. Cognitive Development, 1986; 1(3) 253-271.

[50] Linehan M. (1993). Skills Training Manual for Treating Borderline Personality Disorder. New York: Guilford; 1993.

[51] Clark DM, Bell S, Pape D. An experimental investigation of thought suppression. Behaviour Research \& Therapy,1991; 29(3) 253-257.

[52] Ale CM, Krackow E. Concurrent treatment of early childhood OCD and ODD: A case illustration. Clinical Case Studies, 2011; 10(4) 312-323.

[53] Freeman JB, Garcia AM. Family based treatment for young children with OCD: Therapist guide. New York: Oxford University Press; 2009.

[54] . Abramowitz JS, Taylor S, McKay D. Potentials and limitations of cognitive treatments for obsessive-compulsive disorder. Cognitive Behaviour Therapy, 2005; 34(3), 140-147.

[55] . McLean PD, Whittal ML, Thordarson DS, Taylor S, Söchting I, Koch WJ. Cognitive versus behavior therapy in the group treatment of obsessive-compulsive disorder. Journal of Consulting and Clinical Psychology, 2001; 69(2), 205-214.

[56] Olantunji BO, Rosenfield D, Tart CD, Cottraux J, Powers MB, Smits JAJ. Behavioral versus cognitive treatment of obsessive compulsive disorder: An examination of outcome and mediators of change. Journal of Consulting and Clinical Psychology, 2013; 81(3) 415-428. 
[57] Whittal ML, Thordarson DS, McLean PD. Treatment of obsessive-compulsive disorder: Cognitive behavior therapy vs. exposure and response prevention. Behaviour Research and Therapy, 2005; 43 1559-1576. 
